\title{
Functional Properties of Teff and Oat Composites
}

\author{
George E. Inglett, Diejun Chen, Sean X. Liu \\ Functional Foods Research Unit, National Center for Agricultural Utilization Research, Agricultural Research \\ Service, United States Department of Agriculture, Peoria, USA \\ Email: George.Inglett@ars.usda.gov
}

Received 23 October 2015; accepted 20 December 2015; published 23 December 2015

Copyright (C) 2015 by authors and Scientific Research Publishing Inc.

This work is licensed under the Creative Commons Attribution International License (CC BY).

http://creativecommons.org/licenses/by/4.0/

(c) (i) Open Access

\begin{abstract}
Teff-oat composites were developed using gluten free teff flour containing essential amino acids and minerals along with oat products containing $\beta$-glucan known for lowering blood cholesterol. Teff-oat composites were evaluated for their pasting and rheological properties by a Rapid Visco Analyzer (RVA) and an advanced rheometer. All teff-oat composites showed increased water holding and pasting viscosities with increasing oat contents compared to wheat flour. However, they were only significantly influenced by $80 \%$ oat products in teff-oat composites compared with teff flour alone. OBC (oat bran concentrate) had the highest elastic modulus G' among the starting materials. The elastic modulus G' for teff-Nutrim (oat bran hydrocolloid) composites were decreased with increasing Nutrim contents in composites. In contrast, the increasing content of OBC in composites significantly raised both G' and G". The elastic modulus G' and viscous modulus G" for all teff-OBC composites were higher than teff and wheat flour. All WOF composites showed similar rheological properties. All composites had shear thinning properties that are important to mouthfeel and industrial applications. These teff-oat composites were developed using feasible procedures. They have improved nutritional value and texture qualities for functional food applications.
\end{abstract}

\section{Keywords}

Teff, Oat, $\beta$-Glucan, Gluten-Free, Pasting, Rheology

\section{Introduction}

Teff (Eragrostis tef) is an important ancient grain finding resurgence in the modern age. The attention to teff has been recently increased because of its health benefits. Teff has an attractive nutritional profile having significant 
levels of minerals including calcium, iron, magnesium, phosphorus, potassium, and zinc. Also, teff is rich in vitamins, such as thiamin (B1), riboflavin (B2), vitamin A and K [1]. Furthermore, teff is high in proteins including all 8 essential amino acids that is superior in lysine than wheat or barley along with its high carbohydrates and fiber contents [2]. Teff is beneficial for those who are lactose intolerant since it is gluten-free.

In Egypt, red teff grains are greatly recommended for the improvement of osteoporosis and bone healing conditions. A chemical study of the red teff seeds reported the isolation of seven compounds from its ethanol extract, namely $\beta$-sitosterol (1), $\beta$-amyrin-3-O-(2'-acetyl)-glucoside (2), $\beta$-sitosterol-3- $O$ - $\beta$-D-glucoside (3), naringenin (4), naringenin-4'-methoxy-7-O- $\alpha$-L-rhamnoside (5), eriodictyol-30,7-dimethoxy-4'-O- $\beta$-D-glucoside (6) and isorhamnetin-3-O-rhamnoglucoside (7). This was the first report on the isolation of compounds (2) and (6) in nature. A proximate analysis revealed the high nutritive value of the seeds: carbohydrates (57.27\%), protein (20.9\%), essential amino acids (8.15\%) with major leucine and lysine (1.71\% and 1.35\%, respectively), vitamin B1 (1.56 mg/100g), potassium, and calcium. The seeds yielded 22\% w/w of oil containing $72.46 \%$ of unsaturated fatty acids in which oleic acid was predominant (32.41\%) following by linolenic acid (23.83\%). The ethanolic extracted oil of the seeds exhibited anti-hyperlipedaemic and antihyperglycaemic activities. Oral administration of oil for 10 days resulted in a rise in serum calcium levels in rats [2].

Although teff has excellent nutritional value, it has low pasting viscosity and water holding capacity. In contrast, some oat products have high viscosities and water holding capacities. Also, oat products, such as oat hydrocolloid (Nutrim), oat bran concentrate (OBC), and whole oat flour (WOF), contain $\beta$-glucan that has beneficial health effects on reducing serum cholesterol and postprandial serum glucose levels [3]. In addition, the phenolic and other antioxidant compounds in oat provide health benefits [4]. Oat hydrocolloid products containing $\beta$-glucan have numerous functional food applications to reduce fat content and calories in a variety of foods [5]; control the rheology and texture of food products [6]; modify starch gelatinization and retrogradation [7] [8]; and also provide freezing/thawing stability [9].

Therefore, teff flour and oat products complement each other in nutritional and textural qualities. There are some food products containing teff and oat, such as bread and cookies. However, teff and oat composites were not scientifically studied and reported. Thus, Nutrim, OBC, and WOF were used in this study to produce unique composites containing $\beta$-glucan in combination with teff's distinctive protein and other nutritional components along with its gluten free quality. The objective of our study was to find a feasible way to develop teff-oat composites and to evaluate their functional properties including water holding capacities, pasting, and rheological properties. The functional properties of teff-oat composites could provide useful information for future functional food product developments.

\section{Materials and Methods}

\subsection{Preparation of Teff-Oat Composites}

Teff flour was purchased from Bob’s Red Mill, Milwaukie, OR, USA. OBC (oat bran concentrate) was supplied by Quaker Oats, Chicago, IL, USA. Nutrim (oat bran hydrocolloid, 15 g/100g $\beta$-glucan) was provided by VDF FutureCeuticals (Momence, IL, USA). Nutrim was prepared by steam jet-cooking OBC, sieving, and drumdrying [10] [11]. Organic whole oat flour colloidal fine (WOF) was provided by Grain Millers (Eugene, OR, USA).

Teff flour was mixed with corresponding oat products separately using a mixer (KitchenAid, St Joseph, MI, USA) for 2 min. The mixtures were passed through a 20 mesh sieve followed by additional mixing for 1 min to obtain the desired consistency.

\subsection{Water Holding Capacity}

The water holding capacity (WHC) of the samples was determined according to a previous procedure with minor modifications [12]. Each sample (2 g, dry weight) was mixed with $25 \mathrm{~g}$ of distilled water and vigorously mixed for $1 \mathrm{~min}$ to a homogenous suspension using a Vortex stirrer, held for $2 \mathrm{~h}$, and centrifuged at $1590 \mathrm{~g}$ for 10 min. Each treatment was replicated twice. Water capacity was calculated by the difference between the weight of water added and decanted on dry basis ( $\mathrm{g}$ of water absorbed/100g of dry sample).

\subsection{Pasting Property Measurement}

The pasting properties of samples were evaluated using a Rapid Visco Analyzer (RVA-4, Perten Scientific, 
Springfield, IL). Samples (2.24 g, dry basis) were made up to a total weight of $28 \mathrm{~g}$ with distilled water in a RVA canister ( $8 \%$ solids, w/w). The viscosity of the suspensions was monitored during the following heating and cooling stages. Suspensions were equilibrated at $50^{\circ} \mathrm{C}$ for $1 \mathrm{~min}$, heated to $95^{\circ} \mathrm{C}$ at a rate of $6.0^{\circ} \mathrm{C} / \mathrm{min}$, maintained at $95^{\circ} \mathrm{C}$ for $5 \mathrm{~min}$, cooled to $50^{\circ} \mathrm{C}$ at rate of $6.0^{\circ} \mathrm{C} / \mathrm{min}$, and held at $50^{\circ} \mathrm{C}$ for $2 \mathrm{~min}$. For all test measurements, a constant paddle rotating speed $(160 \mathrm{rpm})$ was used throughout the entire analysis except for 920 rpm in the first $10 \mathrm{~s}$ to disperse sample. Each sample was analyzed in duplicate. The results were expressed in Rapid Visco Analyser units (RVU, 1 RVU = 12 centipoises).

\subsection{Rheological Measurement}

After samples from the RVA were cooled to $25^{\circ} \mathrm{C}$ and equilibrated, they were loaded on a rheometer (AR 2000, TA Instruments, New Castle, DE, USA) using a $4 \mathrm{~cm}$ diameter parallel stainless plate with $1 \mathrm{~mm}$ gap to the surface. The outer edge of the plate was sealed with a thin layer of mineral oil (Sigma Chemical Co., St Louis, MO, USA) to prevent dehydration during the test. All rheological measurements were carried out at $25^{\circ} \mathrm{C}$ using a circulation system within $\pm 0.1^{\circ} \mathrm{C}$. A strain sweep experiment was conducted initially to determine the limits of linear viscoelasticity; then a frequency sweep test was carried out to obtain storage modulus (G') and loss modulus (G”) at frequencies ranging from 0.1 to $10 \mathrm{rad} \mathrm{s}^{-1}$. A strain of $0.5 \%$, which was within the linear viscoelastic range, was used for the dynamic experiments. The steady shear viscosity of the paste was measured as a function of shear rates from 1 to $100 \mathrm{~s}^{-1}$. The steady shear measurements apply varying steady shear deformation on sample material, with magnitude of each deformation depending on specified shear rates. All rheological measurements for samples were performed in duplicate.

\subsection{Statistical Analysis}

All data from replicated samples were analyzed with analysis of variance using Duncan's multiple comparison to determine significant differences $(P<0.05)$ between treatments [13].

\section{Results and Discussion}

\subsection{Compositions of Teff, Oat, and Other Cereals}

Teff has endured the ages from early civilizations as an important food of a nutritious gluten-free source. Both teff and oat are high in proteins, sugar, calcium, iron, magnesium, phosphorus, and zinc. In addition, teff and oat are rich in vitamins. Teff contains the highest potassium and vitamin B2 whereas oat has the highest thiamin (B1) among all products in Table 1. All B vitamins help the body to convert carbohydrates into energy (glucose). Vitamin B2 is important for normal vision along with other nutrients. Some early evidence shows that Vitamin B2 (riboflavin) might help prevent cataracts which can lead to cloudy vision [14]. Vitamin B1 helps the body metabolizes fat and protein and is required for healthy skin, hair, eyes, and liver. It also helps the nervous system function properly and good brain functions [14]. Remarkably, only teff and oat contain vitamin K among all products in Table 1 . Vitamin $\mathrm{K}$ is a fat-soluble vitamin that the body stores it in fat tissue and the liver. It is best known for its role in helping blood clot and bone health [14]. Teff has endured the ages from early civilizations as an important highly nutritious food source with gluten-free quality. Oat was selected in this study because of its $\beta$-glucan content that is helpful for lowering blood cholesterol and improving food texture. Moreover, oat has high quality lipids including monounsaturated and polyunsaturated fatty acid contents. Therefore, the nutritional value and physical properties of gluten free products could be improved by adding the ancient grain teff and oat products to recipes.

\subsection{Water Holding Capacities}

Nutrim had the highest water holding capacity (602.99 g/100g) while wheat flour had the lowest water holding capacity (93.80 g/100g) among all the starting materials (Table 2 \& Figure 1). Nutrim was produced by jetcooking technology using thermal-shearing forces to promote molecular breakdown that probably contributed to its increased water holding capacity [10] [15]. The $\beta$-glucan contents for wheat flour, WOF, OBC, and Nutrim were 1.2 g/100g, 3.9 - 7.47 g/100g, 12.0 g/100g and 15.5 g/100g, respectively. The trend of wheat flour, WOF, OBC, and Nutrim water holding capacity (93.80 g/100g, 158.18 g/100g, 339.52 g/100g, 602.99 g/100g) (Table 2) appeared to be related to their $\beta$-glucan contents, suggesting $\beta$-glucan may be an important factor for WHC. 
Table 1. Compositions of Teff, oat, and other cereals.

\begin{tabular}{|c|c|c|c|c|c|c|}
\hline Nutrient (per 100 g) & Unit & teff & rice & corn & whole wheat & Oat \\
\hline \multicolumn{7}{|l|}{ Proximates } \\
\hline Water & g & 8.82 & 11.89 & 10.37 & 10.74 & 9.37 \\
\hline Energy & kcal & 367 & 366 & 365 & 340 & 371 \\
\hline Protein & g & 13.3 & 5.95 & 9.42 & 13.21 & 13.7 \\
\hline Total lipid (fat) & g & 2.38 & 1.42 & 4.74 & 2.5 & 6.87 \\
\hline Carbohydrate, by difference & g & 73.13 & 80.13 & 74.26 & 71.97 & 68.18 \\
\hline Fiber, total dietary & g & 8 & 2.4 & 7.3 & 10.7 & 9.4 \\
\hline Sugars, total & g & 1.84 & 0.12 & 0.64 & 0.41 & 1.42 \\
\hline \multicolumn{7}{|l|}{ Minerals } \\
\hline Calcium, Ca & $\mathrm{mg}$ & 180 & 10 & 7 & 34 & 47 \\
\hline Iron, Fe & $\mathrm{mg}$ & 7.63 & 0.35 & 2.71 & 3.6 & 4.64 \\
\hline Magnesium, Mg & $\mathrm{mg}$ & 184 & 35 & 127 & 137 & 270 \\
\hline Phosphorus, $\mathrm{P}$ & $\mathrm{mg}$ & 429 & 98 & 210 & 357 & 458 \\
\hline Potassium, K & $\mathrm{mg}$ & 427 & 76 & 287 & 363 & 358 \\
\hline Sodium, Na & $\mathrm{mg}$ & 12 & - & 35 & 2 & 3 \\
\hline Zinc, Zn & $\mathrm{mg}$ & 3.63 & 0.8 & 2.21 & 2.6 & 3.2 \\
\hline \multicolumn{7}{|l|}{ Vitamins } \\
\hline Thiamin (vitamin B1) & $\mathrm{mg}$ & 0.39 & 0.138 & 0.385 & 0.502 & 0.54 \\
\hline Riboflavin (vitamin B2 & $\mathrm{mg}$ & 0.27 & 0.021 & 0.201 & 0.165 & 0.12 \\
\hline Niacin & $\mathrm{mg}$ & 3.363 & 2.59 & 3.627 & 4.957 & 0.82 \\
\hline Vitamin B-6 & $\mathrm{mg}$ & 0.482 & 0.436 & 0.622 & 0.407 & 0.1 \\
\hline Folate, DFE & $\mu g$ & & 4 & 19 & 44 & 32 \\
\hline Vitamin B-12 & $\mu g$ & - & - & - & - & - \\
\hline Vitamin A, RAE & $\mu g$ & - & - & 11 & - & - \\
\hline Vitamin A, IU & $\mathrm{IU}$ & 9 & - & 214 & 9 & - \\
\hline Vitamin E (alpha-tocopherol) & $\mathrm{mg}$ & 0.08 & 0.11 & 0.49 & 0.71 & 0.7 \\
\hline Vitamin K (phylloquinone) & $\mu \mathrm{g}$ & 1.9 & - & - & - & 3.2 \\
\hline \multicolumn{7}{|l|}{ Lipids } \\
\hline Fatty acids, total saturated & g & 0.449 & 0.386 & 0.667 & 0.43 & 1.11 \\
\hline Fatty acids, total monounsaturated & g & 0.589 & 0.442 & 1.251 & 0.283 & 1.98 \\
\hline Fatty acids, total polyunsaturated & g & 1.071 & 0.379 & 2.163 & 1.167 & 2.3 \\
\hline
\end{tabular}

Data were selected from USDA nutrient data base. Teff: uncooked; rice: white, flour; corn: grain, yellow; whole wheat: whole wheat grain flour; oat: Quick Oats, dry.

At ratios 3:2 and 1:4, the WHC of teff-Nutrim composites (165.78/100g, 295.59 g/100g) were higher than that of teff-OBC composites $(160.51 \mathrm{~g} / 100 \mathrm{~g}, 196.59 \mathrm{~g} / 100 \mathrm{~g})$ (Table 2 \& Figure 1). In contrast, the WHC values of teff-Nutrim composites $(135.27 \mathrm{~g} / 100 \mathrm{~g}, 137.57 \mathrm{~g} / 100 \mathrm{~g})$ were slightly higher than those of corresponding teffOBC composites (139.26 g/100g, $154.43 \mathrm{~g} / 100 \mathrm{~g})$ at ratios 4:1 and 3:2. The WHC of teff-WOF composites were the lowest (137.70 g/100g, $138.56 \mathrm{~g} / 100 \mathrm{~g}, 139.47 \mathrm{~g} / 100 \mathrm{~g}$ ) compared to teff-Nutrim and teff-OBC composites at all ratios.

The actual WHC values of teff-OBC and teff-WOF composites are more close to theoretical WHC values 
Table 2. Water holding capacities of starting materials and teff-oat composites.

\begin{tabular}{cccc}
\hline Sample & Actual WHC \% & Theoretical WHC \% & Difference \% \\
\hline teff & $134.92 \pm 1.01 \mathrm{~h}$ & & \\
Nutrim & $602.99 \pm 6.13 \mathrm{a}$ & & \\
OBC & $339.52 \pm 0.85 \mathrm{~b}$ & & 93.26 \\
WOF & $158.18 \pm 0.36 \mathrm{f}$ & 184.58 \\
wheat flour & $93.80 \pm 0.55 \mathrm{i}$ & 249.99 \\
teff:Nutrim 4:1 & $135.27 \pm 0.27 \mathrm{~h}$ & 228.53 & 213.79 \\
teff:Nutrim 3:2 & $137.57 \pm 1.95 \mathrm{~h}$ & 322.15 & 36.58 \\
teff:Nutrim 2:3 & $165.78 \pm 2.08 \mathrm{e}$ & 415.76 & 62.33 \\
teff:Nutrim 1:4 & $295.59 \pm 7.64 \mathrm{c}$ & 175.84 & 97.17 \\
teff:OBC 4:1 & $139.26 \pm 0.67 \mathrm{~h}$ & 216.76 & 102.01 \\
teff:OBC 3:2 & $154.43 \pm 8.10 \mathrm{fg}$ & 257.68 & 1.87 \\
teff:OBC 2:3 & $160.51 \pm 7.48 \mathrm{ef}$ & 298.60 & 5.67 \\
teff:OBC 1:4 & $196.59 \pm 2.36 \mathrm{~d}$ & 139.57 & 9.41 \\
teff:WOF 4:1 & $137.70 \pm 1.80 \mathrm{~h}$ & 144.22 & 2.46 \\
teff:WOF 3:2 & $138.56 \pm 2.05 \mathrm{~h}$ & 148.88 & 153.53 \\
teff:WOF 2:3 & $139.47 \pm 1.57 \mathrm{~h}$ & & \\
teff:WOF 1:4 & $151.07 \pm 0.92 \mathrm{~g}$ & & \\
\hline
\end{tabular}

Means \pm standard deviation; $n=3$; means followed by the same letter within the same column are not significantly different $(P>0.05)$.

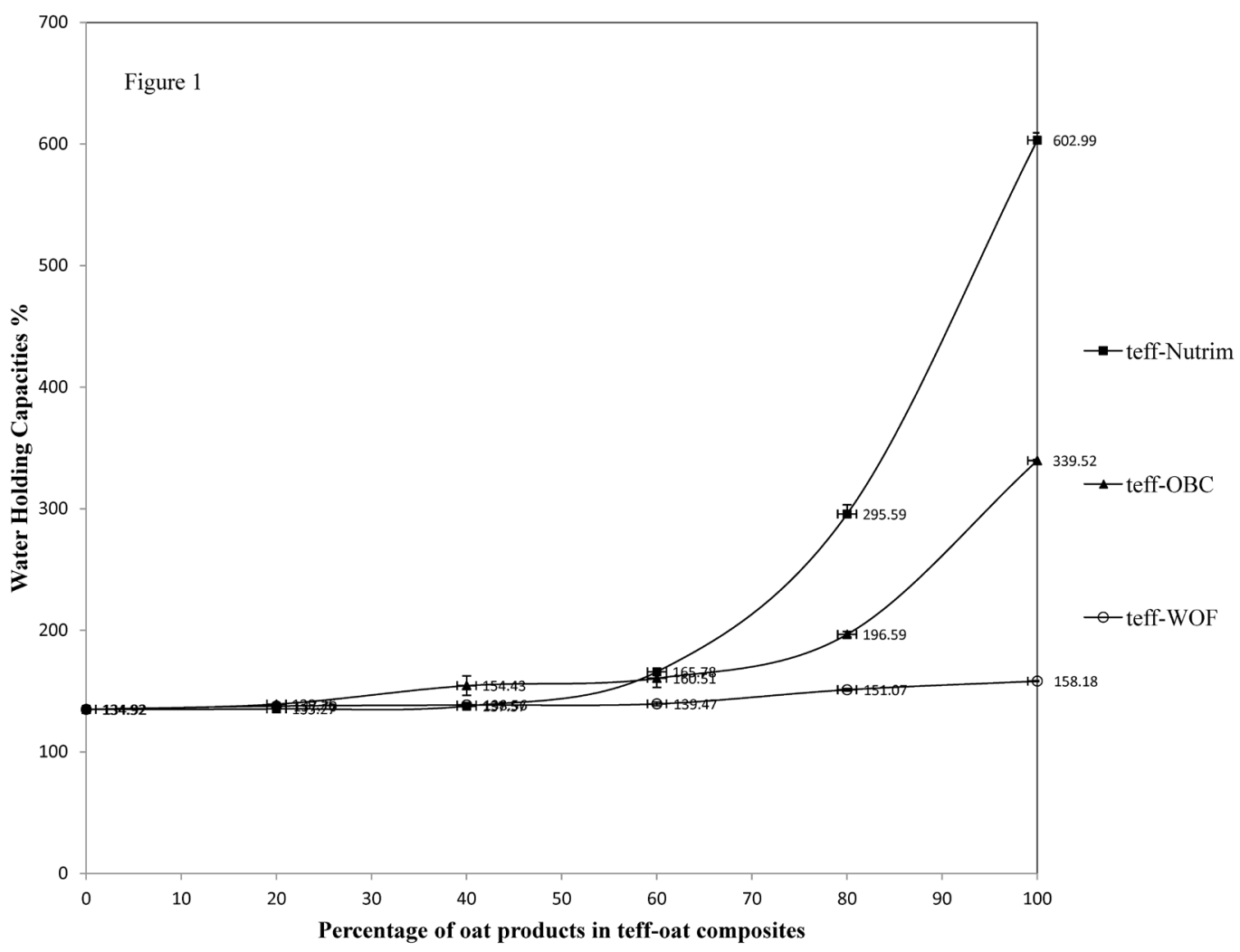

Figure 1. Water holding capacities of starting materials and teff-oat composites. 
(calculated using the actual WHC values of the starting materials) than teff-Nutrim composites (Table 2). In contrast, the actual WHC of teff-Nutrim composites were $100 \%$ - 200\% lower than the theoretical WHC as calculated using the WHC of starting materials (Table 2). Teff flour and oat contain about 13\% protein, and teff is rich in calcium and magnesium (Table 1). It is possible that the calcium and magnesium in teff reacted with protein causing precipitation reducing WHC. It could be a similar mechanism to tofu preparation by coagulating proteins in soymilk with calcium or magnesium sulfate. The proteins coagulate when bonding occurs between the positively charged calcium ions and negatively charged anionic groups of the protein molecules. That reaction resulted in protein clumping and the removal of insoluble material from solution. Nutrim was produced by jet-cooking that may possibly produce anionic groups on the surface that could allow interactions with protein and calcium in teff. The data clearly showed that the teff-Nutrim composite with $20 \%$ Nutrim resulted in a significant reduction (93\%) in WHC. However, the WHC was not decreased linearly when more than 20\% Nutrim was replaced for teff (Figure 1). Those results suggested an interaction between Nutrim and teff. The interaction of teff with Nutrim appeared to reach equilibrium after $20 \%$ teff was added suggesting a possible saturated surface area.

In general, the WHC for teff-oat composites were all higher than teff flour or whole wheat flour alone. Also, the WHC of teff-oat composites were all apparently increased with the higher oat content (Table 2). Significant increases were found for all teff-oat products when the ratios of teff and oat were changed from 2:3 to 1:4.

Teff-oat composites could be widely used in different applications in the food industry because of improved WHC compared to teff or wheat flour alone. Also, they are notable for their thickening properties, syneresis control, and emulsion stabilization along with nutrients in teff.

\subsection{RVA Pasting Properties of Composites}

The pasting curves of all starting materials showed dissimilar patterns (Figure 2(a)). The Nutrim pasting viscosity curve increased sharply ( 23 RVU/min) and showed a significantly high initial peak ( 250 RVU) at $11 \mathrm{~min}$ $\left(90^{\circ} \mathrm{C}\right)$, followed by a rapid decrease in viscosity to $\sim 25 \mathrm{RVU}$ during continued heating. The Nutrim viscosity slightly increased during cooling showing a considerably low final viscosity ( $\sim 58 \mathrm{RVU})$ that was slightly higher than teff flour ( $\sim 50 \mathrm{RVU})$. It is known that the viscosity of a completely gelatinized starch slurry decreases during heating [16]. These characteristics are common for pregelatinized flour [17] and typical for Nutrim since it had undergone jet-cooking during preparation where starch gelatinization occurred. The viscosity of OBC increased gradually ( $\sim 7 \mathrm{RUV} / \mathrm{min})$ to the initial peak ( $\sim 100 \mathrm{RVU})$ at $95^{\circ} \mathrm{C}$, remained almost constant viscosity during continued heating and shearing, and then increased sharply ( $\sim 10 \mathrm{RVU} / \mathrm{min}$ ) resulting in a considerably high final viscosity ( 210 RVU) during cooling. This high viscosity could be due to starch gelatinization and interaction with $\beta$-glucan that resulted in an entanglement of molecules during cooling to form a matrix with greater stability under heating and shearing. WOF had a lower initial viscosity peak ( 50 RVU) than Nutrim and $\mathrm{OBC}$ at $95^{\circ} \mathrm{C}$, showing a small breakdown (peak viscosity minus the lowest point of viscosity after peak), and then slowly increased to a final viscosity ( $\sim 89$ RVU) that was lower than OBC but higher than the rest of the starting materials. The viscosity of teff showed a lower initial peak ( 26 RVU) than all oat products but slightly higher than wheat flour ( $16 \mathrm{RVU})$, and remained constant during heating and shearing, and reached a final viscosity ( $\sim 50$ RVU) similar to Nutrim during cooling. The wheat flour showed the lowest initial peak ( 16 RVU) and final peak ( 17 RVU) among all samples. The trend of initial peaks from wheat flour ( 16 RVU), WOF ( $\sim 8$ RVU), OBC ( 100 RVU), and Nutrim ( $\sim 250 \mathrm{RVU})$ appeared to be related to their $\beta$-glucan contents ( $1.2 \mathrm{~g} / 100 \mathrm{~g}, 8 \mathrm{~g} / 100 \mathrm{~g}, 12 \mathrm{~g} / 100 \mathrm{~g}$ and $15 \mathrm{~g} / 100 \mathrm{~g})$ with the same trends as their water holding capacities.

The initial and final viscosity peaks of teff-Nutrim 4:1 ( 16 RVU), 3:2 ( 20RVU), and 2:3 ( 23 RVU) composites were increased gradually, and slightly lower than teff flour ( 26 RVU, Figure 2(b)). Although the initial peak viscosity of teff-Nutrim 1:4 ( 46 RVU) was significantly higher than the other teff-Nutrim composites and teff, it is much lower than Nutrim ( 250 RVU). A similar trend was observed for teff-OBC composites as teffNutrim composites (Figure 2(c)). It suggested possible interactions between teff with Nutrim and OBC as showed for WHC. Teff-OBC 1:4 had significantly higher final viscosities than the other teff-OBC composites and teff suggesting great stability for the products requiring heating and shearing. All teff-WOF composites had higher initial peaks and final peaks compared to teff and wheat flour (Figure 2(d)). In general, the initial and final peak viscosities of all teff-WOF composites apparently increased with increasing amount of oat products in 


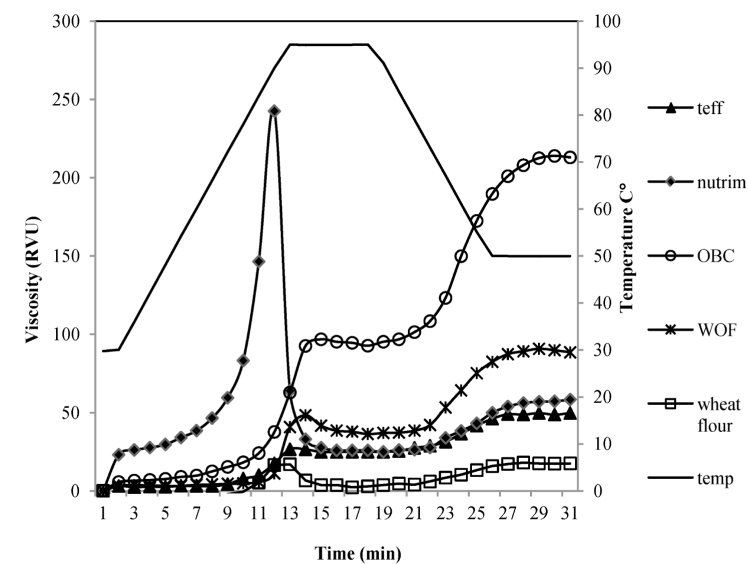

(a)

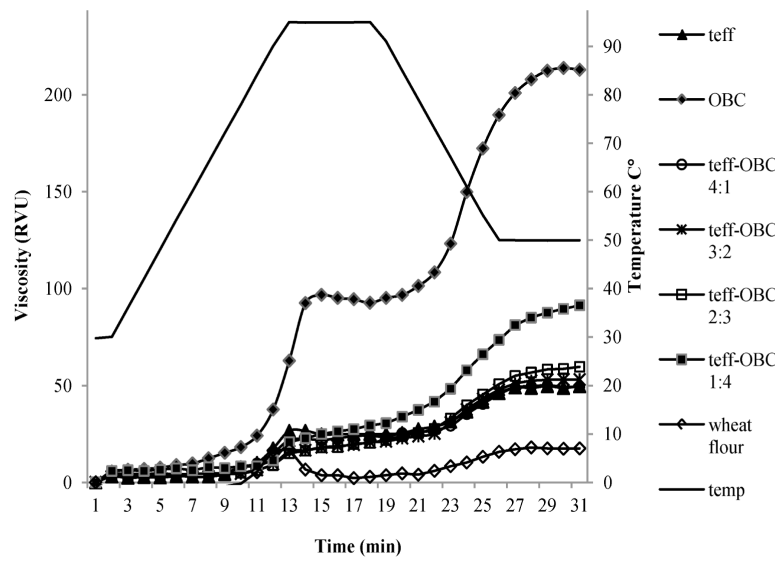

(c)

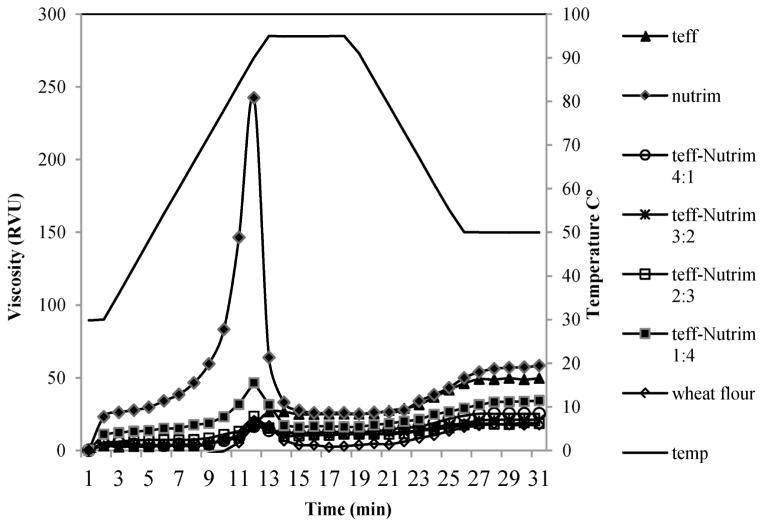

(b)

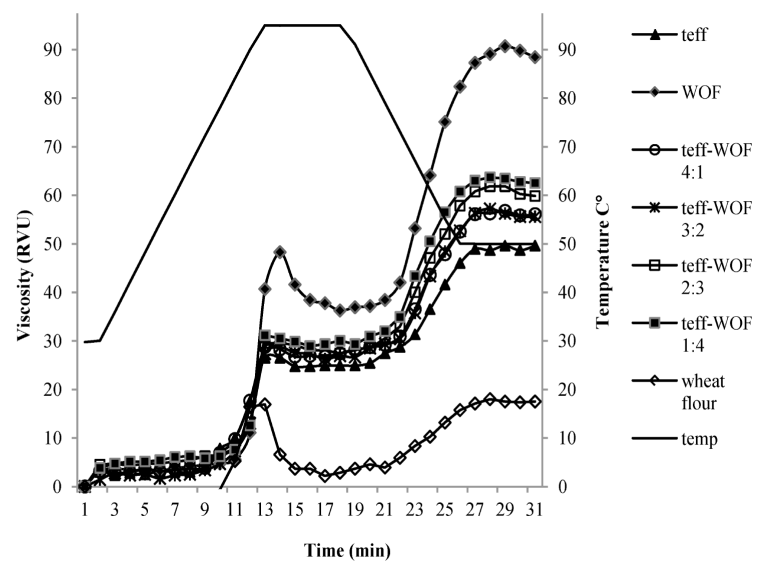

(d)

Figure 2. Rapid Visco-analyser pasting curve of starting ingredients and teff-oat composites. (a) Starting materials; (b) teff -Nutrim composites; (c) teff -OBC composites; (d) teff -WOF composites.

teff-WOF composites. Overall, all teff-oat composites had higher initial and final viscosities than wheat flour. It is probably attributed to the high protein content (30 g/100g) of teff and beta-glucan from oat products. Higher initial peak viscosity was related to starch gelatinization whereas higher final peak viscosities suggested stability after heating and shearing. Improvement in the textural properties of food using oat $\beta$-glucan hydrocolloids has been reported [15]. In general, all 20\% teff-oat composites showed a similar viscosity, indicating they would have similar viscosity properties after shearing and heating. The RVA data were useful since it could provide information for food products. Composites having low viscosity may be suitable for products such as drinks or nutritional bars. Composites with high viscosities could be used for products such as breads, muffin, and cookies for improving the texture quality and health benefits.

\subsection{Rheological Properties of Composites}

The elastic (storage) modulus G' and viscous (loss) G” with frequency for all the starting materials are displayed in Figure 3. The dynamic viscoelastic properties have been related to product quality [15]. Rheometric measurements are often performed to establish the elastic properties, such as gel strength and yield value, both important parameters affecting particle carrying ability and spreadability. The G', an elastic (storage) modulus, represents the non-dissipative component of the mechanical properties of a material and reflects its elastic characteristics. The viscous (loss) modulus (G”) characterizes the dissipative part of the mechanical properties and represents the viscous flow of the material. Viscosity is usually meant resistance to flow or thickness whereas elasticity is related to stickiness or structure. 


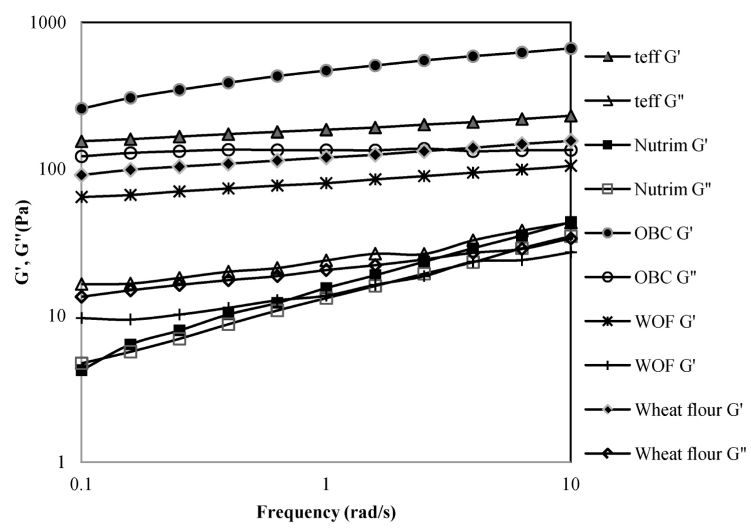

(a)

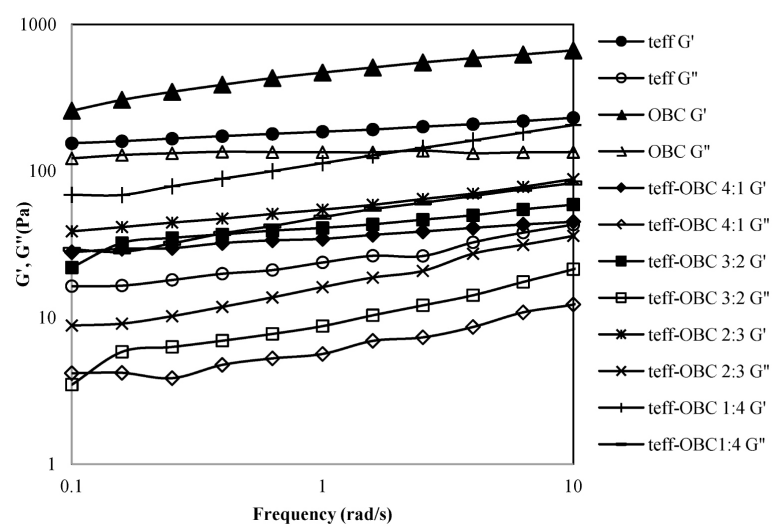

(c)

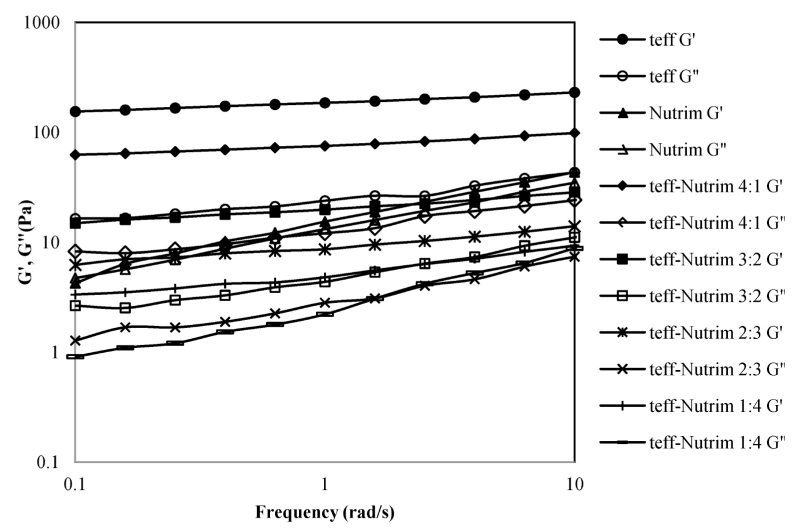

(b)

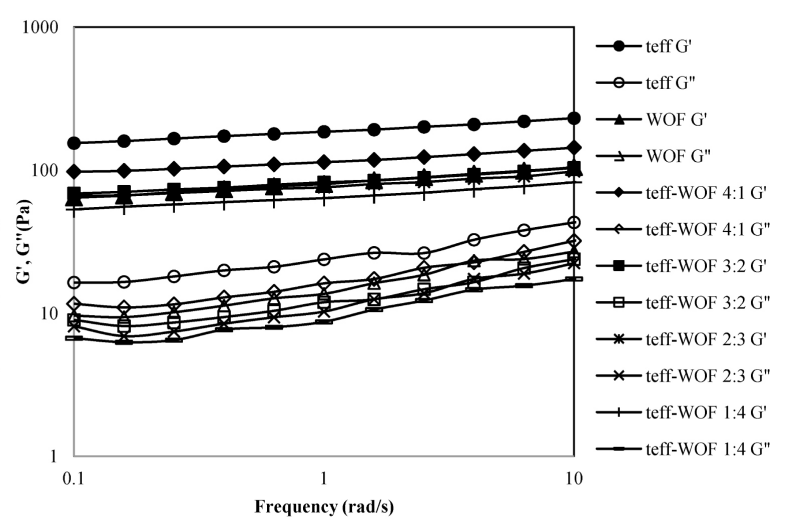

(d)

Figure 3. Dynamic viscoelastic properties of starting materials and teff-oat composites. (a) Starting materials; (b) teffNutrim composites; (c) teff-OBC composites; (d) teff-WOF composites.

OBC had the highest G' and G” moduli that may be contributed to the polysaccharides of OBC including amylose and amylopectin, followed by teff that maybe contributed to its high protein content. Both G' and G" for Nutrim were considerably lower than OBC and WOF perhaps because of their prior hydrothermal processing.

Elastic moduli G' were greater than viscous moduli G” throughout the frequency range for all starting materials with the exclusion of Nutrim (Figure 3(a)). Both moduli were almost frequency-independent throughout the frequency range tested for OBC, teff, WOF, and wheat flour. Also, the large differences between G' and G” were observed indicating that the composite gels could be classified rheologically as elastic gels [15] [18]. In contrast, both moduli G' and G" of Nutrim showed frequency dependence, suggesting colloidal gel properties of Nutrim. In general, a viscoelastic material behaves in a liquid-like manner at low frequencies when the viscoelastic moduli are considered as a function of frequency [19]. The frequency sweep gives information about the gel strength. A steep slope of the G' curve indicates low strength as Nutrim while a slight slope indicates high strength as OBC and teff flour.

The rheological trends of the starting materials were similar to the RVA data (Figure 2(a)). The molecular weight for Nutrim at its peak was $3.0 \times 10^{5}$ that is smaller than OBC $\left(1.5 \times 10^{6}\right)$ as measured by a size-exclusion chromatography instrument (Shiamdzu, VP series, Tokyo, Japan) [20]. Our rheological results showed a maximum value of dynamic storage modulus G' for Nutrim that was lower than OBC. It was in contrast with a previous report that the maximum value of dynamic storage modulus G' decreased with increasing molecular size of the polysaccharides [21]. It may be due to the heating effect on OBC during the RVA test that could change carbohydrate bonds and influence molecular weight.

In general, both moduli (G' and G”) of teff-Nutrim composites are lower than that of teff (Figure 3(b)). The G' of teff-Nutrim 4:1 composites was significantly higher and showed less frequency dependency compared to 
Nutrim and the remaining teff-Nutrim composites. The elastic modulus G' for teff-Nutrim composites were decreased with the increasing Nutrim contents in composites, indicating that the elastic property of these materials were reduced by the addition of Nutrim. The elastic moduli G' of teff-Nutrim composites 2:3 and 1:4 were lower than Nutrim, suggesting interactions between teff and Nutrim. Such results implied that Nutrim contributed a more viscous property whereas teff contributed a more elastic property to the composite systems. All G' and G” from teff-OBC composites were lower than OBC and teff (Figure 3(c)). In contrast to the teff-Nutrim composites, the increasing content of OBC in composites significantly raised both G' and G”. It suggests that the additional OBC increased the elastic properties of teff-OBC composites. The slope of teff-OBC composites 2:3 and 1:4 were increased compared to teff, indicating that the elastic gel property of these materials could be improved by the addition of OBC.

The elastic modulus G' for teff-WOF composite 4:1 was lower than that of teff but higher than WOF while the remaining teff-WOF composites were similar to WOF (Figure 3(d)). Despite the differences in the elastic modulus G' between teff-WOF 4:1 and other teff-WOF composites were noticeable, the viscosity curves of all composites were nearly identical showing similar rheological properties. Also, the elastic or storage modulus G' was greater than loss modulus G” for all teff-WOF composites. It is known that if G' > G”, the material exhibited a solid behavior and the deformation in the linear range will be essentially elastic or recoverable [22].

The rheological results were also explained by $\tan \delta$ values (Figure 4). Loss tangent, $\tan \delta=$ loss modulus G"/storage modulus G', is a dimensionless value that compares the amount of energy lost during a test cycle to the amount of energy stored during this time [19] which indicates whether a material is solid $\left(\delta=0^{\circ}\right)$, liquid $(\delta=$ $\left.90^{\circ}\right)$, or between $\left(0^{\circ}<\delta<90^{\circ}\right)$. The values of $\tan \delta$ are from zero to infinity; and $\tan \delta=1$ means G' $=\mathrm{G}^{\prime \prime}, \tan$ $\delta<1$ represents G' $>$ G”, and $\tan \delta>1$ indicates G' $<$ G”. The loss tangent indicates whether viscous ( $\tan \delta>1)$ or elastic $(\tan \delta<1)$ properties predominate in a sample [23]. The $\tan \delta$ has been used for food products to

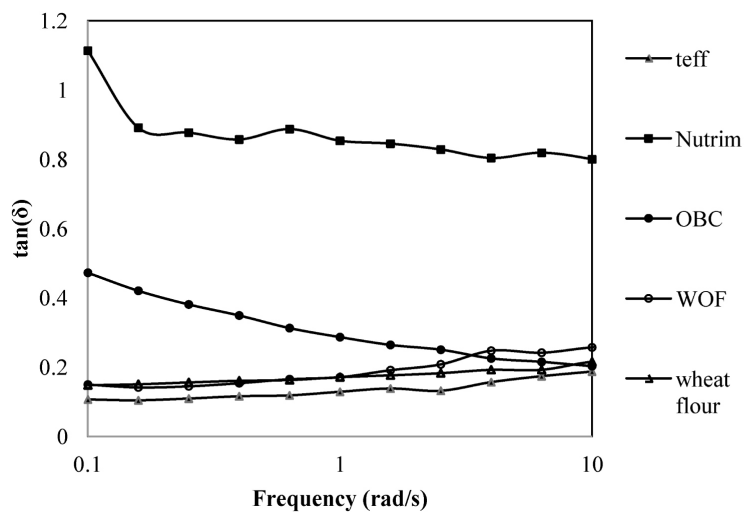

(a)

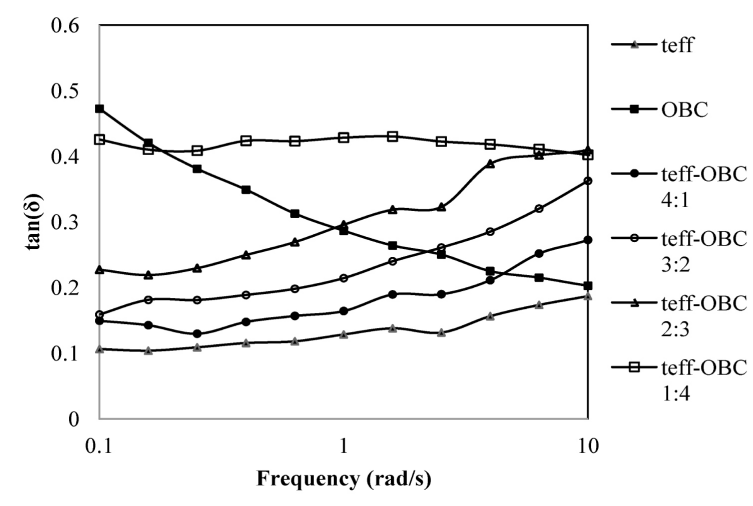

(c)

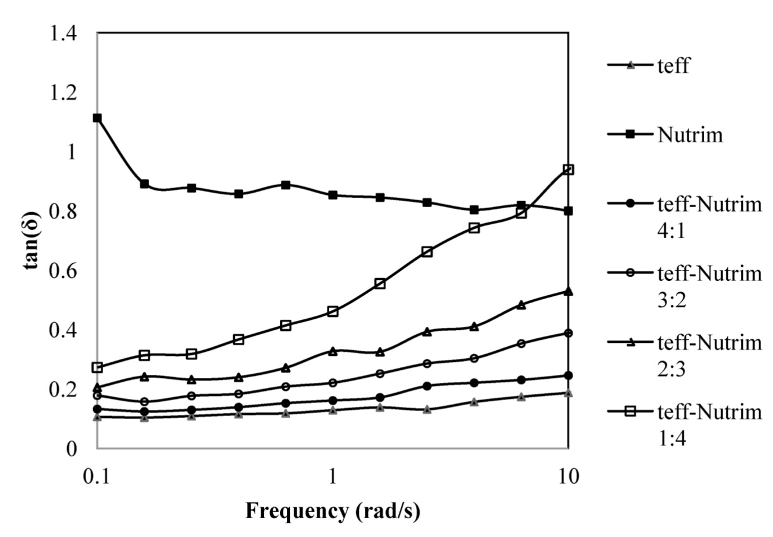

(b)

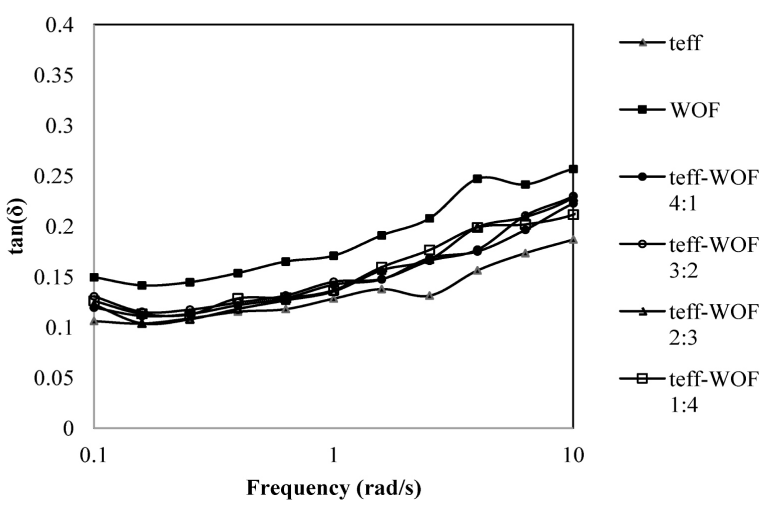

(d)

Figure 4. Curves of $\tan (\delta)$ versus frequency ( $\mathrm{rad} / \mathrm{s}$ ) for starting materials and teff-oat composites. (a) Starting materials; (b) teff-Nutrim composites; (c) teff-OBC composites; (d) teff-WOF composites. 
indicate the strong relationship between the viscous behavior and the degree of hydrolysis such as casein [22]. Of starting materials, Nutrim is the only one having higher $\tan \delta$ value greater than 1 in the beginning and slightly decreased to 0.92 at the end of test indicating more viscous behavior compare with other starting materials (Figure 4(a)). OBC had a lower tan $\delta$ than Nutrim and a decreased trend with an increasing frequency suggesting more elastic properties that may be attributed to some components and structures of OBC (Figure 4(a)). The other starting materials (teff, WOF and wheat flour) had the $\tan \delta$ value less than 1 indicating G' > G" or elastic properties.

Teff-Nutrim composites have $\tan \delta$ values less than one suggesting that the elastic properties predominate. Also, tan $\delta$ values increased from 0.25 to 0.94 at frequency 10 (red/s) as the amount of Nutrim increased from $20 \%$ to $80 \%$ in composites indicating the loss moduli increased with increasing Nutrim contents in composites (Figure 4(b)). The tan $\delta$ values for all teff-OBC composites were higher than that of teff, and lower at low frequency than OBC but higher than OBC at higher frequencies (Figure 4(c)). The $\tan \delta$ is a rheological parameter that shows the contributions of elastic and viscous components to the rheological properties of the materials. Cross points for $\tan \delta$ of teff-OBC composites were observed reflecting possible interactions of teff and OBC. This enhanced elastic property could provide better shape retention during handling and cooking. The $\tan \delta$ curve for teff-OBC 1:4 were nearly flat with frequencies showing great stability and elastic properties of teffOBC. No dramatic changes were observed for WOF and teff-WOF composites indicating that adding WOF to teff had little effect on the viscosity properties of teff (Figure 4(d)).

A viscometric measurement normally consists of a shear rate analysis. The apparent viscosity of the samples vs. shear rate was illustrated in Figure 5. Rheological properties of food products, especially apparent viscosity, have been used as references for predicting their performance during processing [24]. Most food processing and

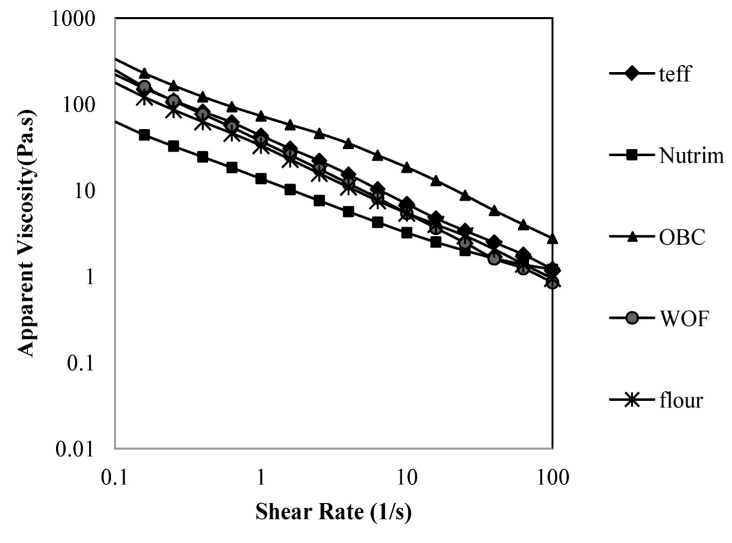

(a)

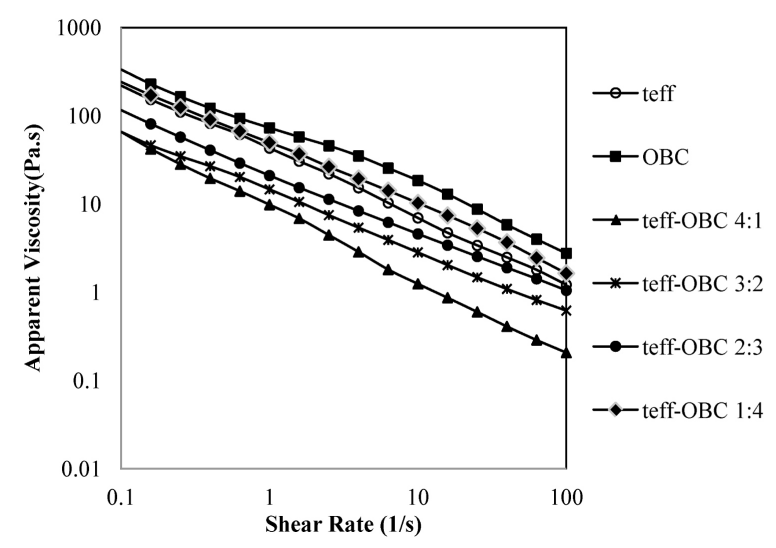

(c)

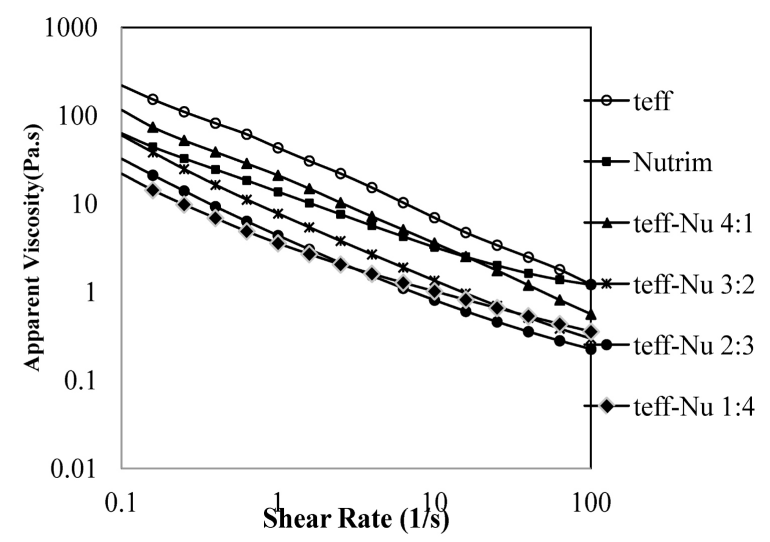

(b)

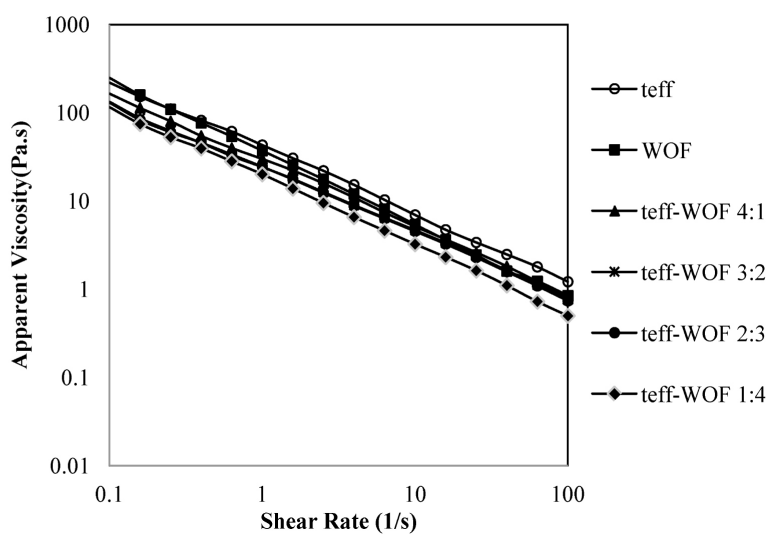

(d)

Figure 5. Apparent viscosities versus shear rates of starting materials and teff-oat composites. (a) Starting materials. (b) teff-Nutrim composites. (c) teff-OBC composites. (d) teff-WOF composites. 
mastication occur in a shear rate range of 1 to $100 / \mathrm{sec}$ as used in this study [25]. All of the samples exhibited shear thinning behaviors over the entire measured shear rates at $25^{\circ} \mathrm{C}$. Shear-thinning behavior can be observed in many food materials such as soy, rice, and wheat flour. This type of shear-thinning behavior is attributed to the disruption of random coil polymers and/or their parallel alignment with flow stream during shearing [26]. OBC seems to have a higher apparent viscosity, followed by teff, WOF, and wheat flour (Figure 5(a)). Interestingly, Nutrim had low apparent viscosity at the low shear rate, and the apparent viscosities increased gradually with increasing shear rate that were higher at high shear rate similar to teff. These properties could be attributed to the beta-glucan component of Nutrim with its high WHC.

The apparent viscosities of teff-Nutrim composites were decreased with the addition of Nutrim to teff, especially at high shear rate (Figure 5(b)). Teff-Nutrim 1:4 composites showed a similar tendency as Nutrim, which has low apparent viscosity at the low shear rate but increased gradually with increasing shear rate, and reached higher apparent viscosities at high shear rate. The apparent viscosities of the teff-OBC composites were increased with the increasing contents of OBC. Similar patterns between teff-OBC composites and teff were observed (Figure 5(c)). A similar trend was observed for WOF composites compared to Nutrim and OBC composites (Figure 5(b) \& Figure 5(d)). The apparent viscosities of teff-WOF composites were not greatly affected by the addition of WOF (Figure 5(d)). The apparent viscosities of WOF composites suggested that those samples could have similar thickening qualities in food processing. Shear-thinning behavior of a material has several potential advantages in food applications since viscosity can be reduced with increasing shear rates. This behavior becomes important in industrial operations such as mixing and pumping. In addition, it was reported that polysaccharide solutions are easily and quickly swallowed where viscosity decreases rapidly at shear rates [27]. Hence, the shear-thinning behavior may contribute a light and nonslimy mouth feel to food products. Since the experimental conditions were similar to actual processing conditions, all our findings on rheological characteristics could be beneficial for processing and developing teff-oat composites for new food applications.

\section{Conclusion}

The pasting and rheological properties of teff-oat composites revealed some useful properties for their potential functional food applications. The teff-oat composites were innovated by a feasible procedure that maintains the original product quality and nutritional values of the starting materials. Teff-oat composites were distinct because the health benefits of the essential amino acids and gluten free uniqueness of teff along with the oat soluble fiber $\beta$-glucan for food texture and coronary heart disease prevention. The composites also have improved water holding capacities, texture, and viscoelastic qualities. The teff-oat composites could be valuable for developing new functional foods having improved nutritional value and desirable texture qualities for health concerned consumers.

\section{References}

[1] USDA Nutrient Database (2014) http://ndb.nal.usda.gov/ndb/search/list http://ndb.nal.usda.gov/ndb/foods/show/6238

[2] Taha, S.E., Shahira M.E. and Amani A.S. (2012) Chemical and Biological Study of the Seeds of Eragrostis tef (Zucc.) Trotter. Natural Product Research: Formerly Natural Product Letters, 26, 619-629. http://dx.doi.org/10.1080/14786419.2010.538924

[3] Klopfenstein, C.F. (1988) The Role of Cereal Beta-Glucans in Nutrition and Health. Cereal Food Worlds, 33, 865-869.

[4] Madhujith, T. and Shahidi, F. (2007) Antioxidative and Antiproliferative Properties of Selected Barley (Hordeum vulgare L.) Cultivars and Their Potential for Inhibition of Low-Density Lipoprotein (LDL) Cholesterol Oxidation. Journal of Agricultural and Food Chemistry, 55, 5018-5024. http://dx.doi.org/10.1021/jf070072a

[5] Lee, S., Inglett, G.E. and Carriere, C.J. (2004) Effect of Nutrim Oat Bran and Flaxseed on Rheological Properties of Cakes. Cereal Chemistry, 81, 637-642. http://dx.doi.org/10.1094/CCHEM.2004.81.5.637

[6] Rosell, C.M., Rojas, J.A. and de Barber, C.B. (2001) Influence of Hydrocolloids on Dough Rheology and Bread Quality. Food Hydrocolloids, 15, 75-81. http://dx.doi.org/10.1016/S0268-005X(00)00054-0

[7] Rojas, J.A., Rosell, C.M. and de Barber, C.B. (1999) Pasting Properties of Different Wheat Flour-Hydrocolloid Systems. Food Hydrocolloids, 13, 27-33. http://dx.doi.org/10.1016/S0268-005X(98)00066-6

[8] Lee, S., Warner, K. and Inglett, G.E. (2005) Rheological Properties and Baking Performance of New Oat $\beta$-GlucanRich Hydrocolloids. Journal of Agricultural and Food Chemistry, 53, 9805-9809. http://dx.doi.org/10.1021/jf0513680 
[9] Lee, M.H., Baek, M.H., Cha, D.S., Park, H.J. and Lim, S.T. (2002) Freeze-Thaw Stabilization of Sweet Potato Starch Gel by Polysaccharide Gums. Food Hydrocolloids, 16, 345-352. http://dx.doi.org/10.1016/S0268-005X(01)00107-2

[10] Inglett, G.E. (2000) Soluble Hydrocolloid Food Additives and Method of Making. U. S. Patent Number 6,060,519.

[11] Inglett, G.E. (2011) Low-Carbohydrate Digestible Hydrocolloidal Fiber Compositions. U. S. Patent Number 7,943,766B2.

[12] Ade-Omowaye, B.I.O., Taiwo, K.A., Eshtiaghi, N.M., Angersbach, A. and Knorr, D. (2003) Comparative Evaluation of the Effects of Pulsed Electric Field and Freezing on Cell Membrane Permeabilisation and Mass Transfer during Dehydration of Red Bell Peppers. Innovative Food Science \& Emerging Technologies, 4, 177-188. http://dx.doi.org/10.1016/S1466-8564(03)00020-1

[13] SAS Institute Inc. (1999) The SAS ${ }^{\circledR}$ System for Windows ${ }^{\circledR}$, Version 8e. Cary, NC.

[14] University of Maryland Medical Center. http://umm.edu/health/medical/altmed/supplement/vitamin-b2-riboflavin\#ixzz3YoxeKZsF

[15] Lee, S. and Inglett, G.E. (2006) Rheological and Physical Evaluation of Jet-Cooked Oat Bran in Low Calorie Cookies. International Journal of Food Science and Technology, 41, 553-559. http://dx.doi.org/10.1111/j.1365-2621.2005.01105.x

[16] Guha, M., Ali, S.Z. and Bhattacharya, S. (1998) Effect of Barrel Temperature and Screw Speed on Rapid Viscoanalyser Pasting Behaviour of Rice Extrudate. International Journal of Food Science and Technology, 3, 259-266. http://dx.doi.org/10.1046/j.1365-2621.1998.00189.x

[17] Lai, H.-M. and Cheng, H.-H. (2004) Properties of Pregelatinized Rice Flour Made by Hot Air or Gum Puffing. International Journal of Food Science and Technology, 39, 201-212. http://dx.doi.org/10.1046/j.0950-5423.2003.00761.x

[18] Lai, L.S. and Liao, C.-L. (2002) Steady and Dynamic Shear Rheological Properties of Starch and Decolorized Hsiantsao Leaf Gum Composite Systems. Cereal Chemistry, 79, 58-63. http://dx.doi.org/10.1094/CCHEM.2002.79.1.58

[19] Ferry, J.D. (1980) Viscoelastic Properties of Polymers. 3rd Edition, John Wiley, New York.

[20] Kim, S., Inglett, G.E. and Liu, S.X. (2008) Content and Molecular Weight Distribution of Oat $\beta$-Glucan in Oatrim, Nutrim, and C-Trim Products. Cereal Chemistry, 85, 701-705. http://dx.doi.org/10.1094/CCHEM-85-5-0701

[21] Vaikousi, H., Biliaderis, C.G. and Izydorczyk, M.S. (2004) Solution Flow Behavior and Gelling Properties of WaterSoluble Barley $(1 \rightarrow 3,1 \rightarrow 4)-\beta$-Glucans Varying in Molecular Size. Journal of Cereal Science, 39, 119-137. http://dx.doi.org/10.1016/j.jcs.2003.09.001

[22] Gravier, N.G., Zaritzky, N.E. and Califano, A.N. (2004) Viscoelastic Behavior of Refrigerated and Frozen LowMoisture Mozzarella Cheese. Journal of Food Science, 9, 123-128. http://dx.doi.org/10.1111/j.1365-2621.2004.tb13364.x

[23] Sutheerawattananonda, M. and Bastian, E.D. (1998) Monitoring Process Cheese Meltability Using Dynamic Stress Rheometry. Journal of Texture Studies, 29, 169-183. http://dx.doi.org/10.1111/j.1745-4603.1998.tb00162.x

[24] Salvador, A., Sanz, T. and Fiszman, S. (2002) Effect of Corn Flour, Salt, and Leavening on the Texture of Fried, Battered Squid Rings. Journal of Food Science, 76, 730-773. http://dx.doi.org/10.1111/j.1365-2621.2002.tb10667.x

[25] Bloksma, A. (1998) Rheology of the Bread Making Process. Paper presented at 8th International Cereal and Bread Congress, Lausanne, PMid: 9772201.

[26] Salamone, J.C. (1996) Polymeric Materials Encyclopedia. CRC Press, Boca Raton.

[27] Szczesniak, A.S. and Farkas, E. (1962) Objective Characterization of the Mouthfeel of Gum Solutions. Journal of Food Science, 27, 381-385. http://dx.doi.org/10.1111/j.1365-2621.1962.tb00112.x 